\title{
Osteopontin, CCL5 and CXCL9 are independently associated with psoriasis, regardless of the presence of obesity
}

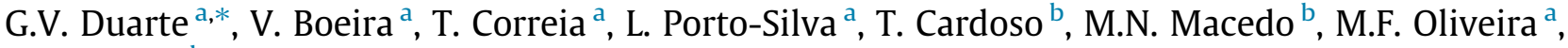 \\ E. Carvalho ${ }^{\mathrm{b}, \mathrm{c}}$
}

a Dermatology Department, Federal University of Bahia, Rua Padre Feijó s/n., Salvador, Bahia 40.110-150, Brazil

b Immunology Department, Federal University of Bahia, Rua Padre Feijó s/n., Salvador, Bahia 40.110-150, Brazil

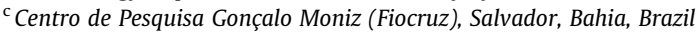

\section{A R T I C L E I N F O}

\section{Article history:}

Received 30 November 2014

Received in revised form 21 April 2015

Accepted 22 April 2015

Available online 9 May 2015

\section{Keywords:}

Severity

Cytokines

Biomarkers

Comorbidities

Obesity

\begin{abstract}
A B S T R A C T
Psoriasis is an autoimmune disease associated with the production of pro-inflammatory cytokines. The identification of these molecules in the pathogenesis of psoriasis facilitated the use of monoclonal antibodies to block their actions as a treatment for severe psoriasis. An increased inflammatory response has been documented in patients with obesity, a condition that is associated with the occurrence and severity of psoriasis. Osteopontin (OPN), TNF and CXCL9 levels are enhanced in patients with psoriasis, although OPN has been documented in the adipose tissue of obese patients without psoriasis. The prevalence of obesity is much higher in psoriasis patients compared with the general population. Thus, we aimed to evaluate the relationship between cytokine levels and psoriasis in the context of obesity. We compared OPN and CXCL9 plasma levels among 117 psoriasis patients and 27 healthy body mass index-matched subjects using ELISA. We also analyzed the TNF, CCL2 and CCL5 levels in a smaller subgroup of patients and matched controls. Median OPN, CCL5 and CXCL9 levels were significantly higher in psoriasis patients compared with the controls, independent of obesity. There was no difference between the median CCL2 levels in the psoriasis patients and the controls $(P<0.05)$, although the CCL2 levels were elevated in obese patients compared with non-obese psoriasis patients $(P<0.001)$. Facial involvement and the psoriasis area severity index (PASI) score were not associated $(P<0.05)$ with OPN levels or elevated levels of chemokines. There was no significant correlation between the OPN and CXCL9 levels or the OPN and TNF levels in psoriasis patients. This work confirms that OPN, CCL5 and CXCL9 plasma levels are higher in psoriasis patients and provides evidence that their higher levels are not a consequence of obesity. Furthermore, the results demonstrate that OPN production is independent of TNF- $\alpha$ and CXCL9.
\end{abstract}

(C) 2015 Elsevier Ltd. All rights reserved.

\section{Introduction}

Osteopontin (OPN) is a glycoprotein produced by immune system cells, adipose tissue macrophages, keratinocytes, hepatocytes, smooth muscle, endothelial cells, and osteoblasts, among others [1]. OPN can interact with different ubiquitously expressed receptors, including integrins and CD44 variants, and acts in physiological and pathological processes, including inflammation and immune responses, enhancing Th1 and inhibiting Th2 responses. OPN also participates in the regulation of Th17 cell lineage and is associated with autoimmune disorders, such as rheumatoid arthritis and Crohn's disease [1]. OPN has two variants: secreted and intracellular. Secreted OPN functions in adhesion, cellular

\footnotetext{
* Corresponding author at: Avenida Tancredo Neves, 620 Sala 417, Caminho das Árvores, Salvador, Bahia, Brazil. Tel./fax: +55 7135650691.

E-mail address: gleisonvduarte@yahoo.com.br (G.V. Duarte).
}

migration, secretion of Th1 cytokines, and prevents apoptosis. Intracellular OPN regulates macrophage migration and secretion of TNF- $\alpha$ from dendritic and plasma cells. Both OPN variants participate in the regulation of the Th17 cell lineage and are associated with autoimmune disorders $[1,2]$. Elevated serum concentrations of OPN were identified in psoriasis patients [2,3] and in the adipose tissue of obese subjects [3] as a result of macrophage accumulation [4]; elevated OPN levels may contribute to obesity-induced inflammation [1]. This could explain why OPN concentrations are not reduced after bariatric surgery weight loss [3-6], why OPN levels in obese individuals do not correlate with body fat percentage, and the high expression of OPN in adipose tissues [7].

Chemokines are often found at higher levels in peripheral blood than cytokines, making them a more convenient and reliable target for quantification [8]. Chemokines are possible therapeutic targets for psoriasis; chemokine inhibition could decrease pro-inflammatory cytokines at an early stage of the disease and 
may be an interesting alternative to specific cytokine blockade, as chemokine receptors have more than one ligand and different chemokines can bind to different receptors. This may favor chemokines and OPN as the most promising biomarker candidates in psoriasis $[2,9]$. The chemokine network of different chronic inflammatory skin diseases, such as psoriasis and atopic dermatitis, reflects the predominant Th polarization. CC chemokines may be associated with eosinophil and Th2 cell chemoattraction in atopic dermatitis, while CXC chemokines associate with neutrophils and Th1 in psoriasis [10]. Psoriasis epidermis lesions express CCL4, CXCL8, CXCL2, CCL2, CXCL9, and CXCL10 [11,12]. CXCL9 is among the most studied chemokines in psoriasis; in keratinocytes, dose-dependent inhibition of CXCL9 transcription occurs after dimethyl fumarate therapy [13] and its levels are consistently higher in psoriasis patients when compared to controls [14,15]. High serum levels of CXCL8, CXCL9, and CXCR3 were also described in psoriasis patients $[12,13]$. The levels of CCL2 and CCL5 decrease after Goeckerman therapy and consequent improvement of skin lesions [16]; nevertheless, this field remains controversial. Recently, a positive correlation between OPN and BMI in obese individuals was observed [17]. Plasma OPN levels are raised in patients with coronary artery disease, hypertension, and diabetes mellitus [18]; chemokines, cytokines, and adipokines are frequently enhanced in obese individuals as well [19]. Chemokines, including CCL2, CCL5, CCL7, CCL8, CXCL5, CXCL8, and CXCL10, are overexpressed in adipose tissue and their serum levels are dramatically increased in obese versus lean individuals [20]. According to Maksymowych et al., the effect of comorbidities on levels of a soluble biomarker is an important source of variability that should be examined [21].

The role of cytokines and chemokines in psoriasis is well-studied, but the relationship between osteopontin, obesity, and psoriasis is not completely understood.

As obesity prevalence is much higher in psoriasis patients $[22,23]$, this prompted us to investigate whether OPN, CXCL9, CCL2, and CCL5 elevation in psoriasis is associated with the presence of obesity. Moreover, we analyzed if these molecules could also be biomarkers of psoriasis severity.

\section{Methods}

\subsection{Patients}

From August 2010 to June 2013, 117 adult ( $\geqslant 18$ year-old) patients who presented plaque psoriasis and 29 body mass index (BMI)-matched controls were admitted to the outpatient Dermatology Clinic of the Federal University of Bahia (Brazil) and invited to participate in the study. The patients had not used any systemic psoriasis drug or been submitted to phototherapy in the preceding 4 weeks. Patients and healthy volunteers signed an informed written consent to participate in this study, which was approved by the Maternidade Climério de Oliveira ethics committee. Clinical parameters were collected, including age, gender, body weight, height, psoriasis severity, waist circumference, facial/nail involvement, duration of disease, age of onset, family history, smoking status, previous and current therapies, and the presence of comorbidities (including hypertension, diabetes, dyslipidemia, depression, psoriatic arthritis (PsA), among others). The psoriasis area severity index (PASI) was used to assess the severity of psoriasis and a score $\geqslant 10$ was considered as moderate-to-severe psoriasis. Facial involvement was also analyzed as a predictor of severity $[24,25]$. Obesity was diagnosed if BMI $\geqslant 30$. Central obesity was considered if waist-to-hip ratio (WHR) $\geqslant 0.9$ in men or $\geqslant 0.85$ in women or waist-circumference $(\mathrm{WC}) \geqslant 102$ or $88 \mathrm{~cm}$, respectively, or if waist-to-height ratio $(\mathrm{RWH}) \geqslant 0.5$ in both genders.

\subsection{Measurement of plasma OPN, TNF- $\alpha$ and chemokines concentrations}

For each participant, $10 \mathrm{~mL}$ of venous blood samples were collected. After $15 \mathrm{~min}$ of centrifugation at $400 \mathrm{~g}$, the plasma was rapidly pipetted off and kept frozen at $-20^{\circ} \mathrm{C}$ until analysis. OPN levels were evaluated by Quantikine ${ }^{\circledR}$ enzyme-linked immunosorbent assay kits purchased from R\&D Systems Europe (product code DOST00, Abingdon, UK). The sensitivity limit of this assay was $0.011 \mathrm{ng} / \mathrm{mL}$. Quantities of OPN in the samples were calculated in comparison to a serial standard, and the results were expressed as ng/mL. CXCL9 was analyzed by a Quantikine ${ }^{\circledR}$ ELISA Human CXCL9/MIG Immunoassay (R\&D, product code DCX900). The detectable CXCL9 ranged from 31.3 to $2000 \mathrm{pg} / \mathrm{mL}$. CCL2 and CCL5 were analyzed by a Human CCL2/MCP-1 Quantikine ${ }^{\circledR}$ ELISA Kit (R\&D, product code DCP00) and a Human CCL5/RANTES Quantikine ${ }^{\circledR}$ ELISA Kit (R\&D, product code DRNOOB), respectively, whose assays' ranges were both $31.2-2000 \mathrm{pg} / \mathrm{mL}$. TNF was analyzed by a Human TNF ELISA Kit Bd OptEIA ${ }^{\text {TM }}$ (BD Biosciences Pharmingen 555212). The detectable TNF- $\alpha$ ranged from 7.8 to $500 \mathrm{pg} / \mathrm{mL}$. The immunoassays were performed according to manufacturer's instructions and a SoftMax ${ }^{\circledR}$ ProV5 spectrometer was used in these assays.

\subsection{Statistical analysis}

A total of 117 plasma samples from psoriasis patients and 27 plasma samples from BMI-matched control subjects were enrolled. Two control subjects were excluded to match the groups by BMI. Osteopontin was determined in all plasma from patients and controls and TNF- $\alpha$ was analyzed in 74 psoriasis patients. For CCL2 and CCL5 levels, we analyzed a subgroup of 31 patients and 9 controls (approximately, $1 / 3$ of patients and $1 / 3$ of controls, selecting the first 31 patients and 9 controls readily available for analysis). In order to reduce the interference of intermediate PASI values in the analyses, besides comparing PASI $\leqslant 10$ versus PASI $>10$, patients were also compared for having PASI values $\leqslant 10$ versus $>15$. Statistical analyses were performed in GraphPad Prism 5 version 5.00 for Windows (GraphPad Software, San Diego, California, USA) and SAS 9.0 ${ }^{\circledR}$ (SAS Institute Inc., Cary, NC, USA). Quantitative variables were analyzed using a Kolmogorov-Smirn ov-Lilliefors normality test. Comparisons between quantitative variables were performed using the Mann-Whitney and KruskalWallis tests. The chi-square test was used to determine the association between two categorical variables. Correlations between non-parametric distribution variables were ascertained using the Spearman test. Analyses of receiver operating characteristics (ROC) curves were performed to calculate the cut-off values of plasma OPN associated with the occurrence of psoriasis, according to the most accurate values obtained. A logistic regression model was conducted to evaluate the association between plasma OPN levels, psoriasis, and psoriasis severity, and between psoriasis and the epidemiological features of the psoriasis patients. Results were considered statistically significant at $P$-values $<0.05$.

\section{Results}

\subsection{Epidemiological features and obesity evaluation in patients with psoriasis and control subjects}

The age, gender, and different measurements of obesity are shown in Table 1. There was a significant difference in age between the two groups. The two groups were comparable regarding obesity evaluation. Thirty-seven patients (31.2\%) presented moderate-to-severe psoriasis (PASI $\geqslant 10$ ). The mean \pm SD PASI 
Table 1

Epidemiologic features and overweight measurement in psoriasis patients and controls.

\begin{tabular}{llll}
\hline Variable & Psoriasis & Controls & $P$-value \\
\hline Gender $($ fem) & $49.5 \%$ & $64.0 \%$ & $P>0.05$ \\
Age $($ years) & $50 \pm 14$ & $39 \pm 14$ & $P<0.05$ \\
BMI $\left(\mathrm{kg} / \mathrm{m}^{2}\right)$ & $28.3 \pm 5.5$ & $30.0 \pm 6.0$ & $P>0.05$ \\
WC $(\mathrm{cm})$ & $95 \pm 13$ & $93 \pm 27$ & $P>0.05$ \\
WHR & $0.9 \pm 0.08$ & $0.7 \pm 0.3$ & $P>0.05$ \\
RWH & $0.58 \pm 0.08$ & $0.57 \pm 0.2$ & $P>0.05$ \\
\hline
\end{tabular}

Legend: BMI - body mass index; WC - waist circumference; WHR - waist-to-hip ratio; $\mathrm{RWH}$ - waist-to-height ratio.

score was $9.0 \pm 8.0$ (range: $0.5-40.9$ ). PsA was diagnosed in 16 (13.6\%) patients. Other comorbidities were diagnosed, including diabetes mellitus ( $12 ; 10 \%)$, hypertension (30;25.6\%), dyslipidemia (25; 21.3\%), alcohol use (63; 53.8\%), smoking (50;42\%), and non-alcoholic fatty liver disease (10; $8.5 \%)$. A family history of psoriasis was present in $48(41 \%)$ patients. Facial and nail involvement were seen in 49 (41\%) and $56(47 \%)$ patients, respectively. PASI scores of patients with facial involvement $(12.1 \pm 9.0)$ were higher than in patients without $(6.8 \pm 6.4, P<0.001)$.

Age and duration of the disease did not present a linear correlation $(P=0.354$; Spearman). Possible medications that could interfere in psoriasis severity were registered as follows: angiotensin convertor inhibitor (three patients), $\beta$-blockers (two patients), and non-steroidal anti-inflammatories (two patients).

\subsection{Levels of OPN in psoriasis and control group}

OPN levels in psoriasis patients (median $44.6 \mathrm{ng} / \mathrm{mL}$ (range 3500)) were significantly higher than those in the healthy control group (median $12.5 \mathrm{ng} / \mathrm{mL}$ (range 2.5-47.5; $P<0.05$ )) (Fig. 1). OPN levels were compared, with controls, between obese and non-obese patients. (Fig. 2); there were no differences between obese and non-obese psoriasis patients, but both groups had statistically higher levels of OPN compared to their related controls. Additionally, in patients with psoriasis, no statistical correlation between BMI and OPN was observed. No differences in OPN levels were found between patients with PASI $<10 \times$ PASI $\geqslant 10$ nor between patients with PASI $<10 \times$ PASI $\geqslant 15$. OPN levels were not associated with the presence of facial involvement or PsA. Central obesity, determined by RHT, WC, and WHR, did not show significant differences in OPN levels. Comparing patients with recently diagnosed psoriasis ( $\leqslant 5$ years) versus long-term psoriasis

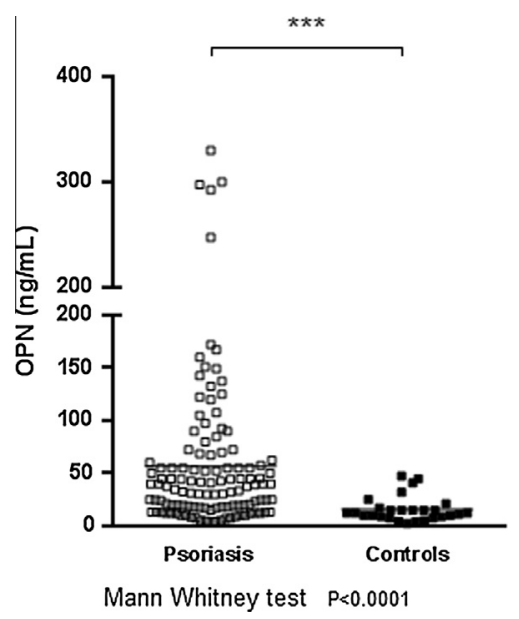

Fig. 1. Comparison of osteopontin (OPN) plasma levels between patients and controls ${ }^{* * *} P<0.0001$.

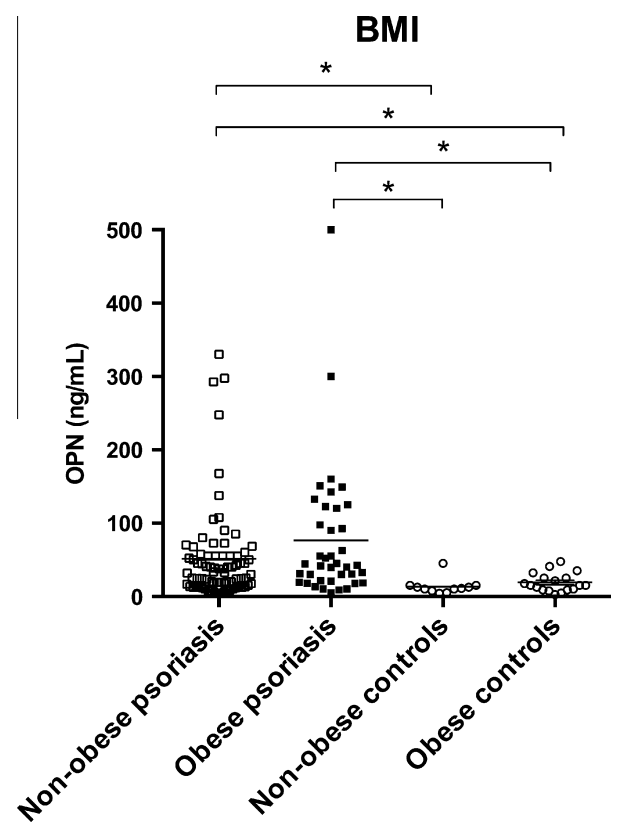

Fig. 2. Comparison of osteopontin (OPN) plasma levels in obese and non-obese patients and controls. ${ }^{*} p<0.05$.

$(\geqslant 15$ years), there were no differences in OPN levels. No other epidemiological variable influenced OPN levels.

Analyses of non-parametric ROC were performed to calculate the most accurate cut-off value of plasma OPN. The area under the curve was 0.795 (95\% confidence interval [CI] 0.712-0.878). The best cut-off value of plasma OPN for the occurrence of psoriasis was $17.65 \mathrm{ng} / \mathrm{mL}$, with $76 \%$ sensitivity and $77 \%$ specificity. A logistic regression model demonstrated that OPN levels above this cut-off were positively associated with psoriasis ( $\beta 1$ value 2.9575; $p<0.001$ ). The occurrence of high OPN plasma levels was associated with an $\mathrm{OR}=19.25$ for the occurrence of psoriasis in relation to healthy individuals (95\% CI 6.82-54.3). Moderate to severe psoriasis was not a predictor of high OPN levels ( $\beta 1$ value $0.2166, P=0.7$ ). Despite age differences among patients and controls, no association between high OPN levels and age or disease duration was found. For multivariate analyses, we chose possible risk factors for high OPN levels (Table 2).

\subsection{Levels of chemokines (CCL2, CCL5 and CXCL9) in psoriasis and control groups}

CCL5, and CXCL9 were significantly higher in psoriasis patients than in controls (Fig. 3). To evaluate if chemokine levels are influenced by psoriasis severity or by obesity, they were compared between patients with PASI $<10$ and PASI $>10$, patients with

Table 2

Multivariate logistic regression analysis of factors associated with high plasma OPN in psoriasis patients.

\begin{tabular}{llcl}
\hline $\begin{array}{l}\text { Inflammatory } \\
\text { mediator }\end{array}$ & Clinical/epidemiological & $\beta$ & $P$-value \\
& variable & & \\
\hline OPN $\geqslant 17.65 \mathrm{ng} / \mathrm{mL}$ & Psoriasis vulgaris & 0.0017 & $P<0.01$ \\
& Severe psoriasis & 0.2166 & 0.70 \\
& PsA diagnosis & 0.4809 & 0.56 \\
& Facial involvement & -0.0152 & 0.97 \\
& Obesity & 0.2470 & 0.67 \\
& WTR $\geqslant 0.5$ & -0.060 & 0.94 \\
& Age & -0.006 & 0.73 \\
& Disease's duration & -0.002 & 0.13 \\
\end{tabular}


PASI $<10$ and PASI $\geqslant 15$, and obese and non-obese patients. No correlation was found between PASI score and OPN $(P=0.4)$ or CXCL9 levels $(P=0.1)$. There were no differences observed between obese and non-obese psoriasis patients, except for CCL2 levels, which were, on average, $690 \mathrm{pg} / \mathrm{mL}(260-4230)$ and $425 \mathrm{pg} / \mathrm{mL}$ (129-3080), respectively $(P<0.001)$. Psoriasis severity did not alter CXCL9, CCL2, or CCL5 levels. Central obesity, determined by RHT, WHR, and WTR, did not show significant differences in chemokine levels in psoriasis patients or in controls. To evaluate if OPN levels were associated with TNF and CXCL9, two known chemokines that are enhanced in psoriasis patients, the values of OPN and TNF and OPN and CXCL9 were plotted in Fig. 4a and b, respectively. OPN levels were not correlated with TNF ( $P=0.68$; Spearman $)$ or with CXCL9 ( $P=0.88$; Spearman).

\section{Discussion}

Pro-inflammatory cytokines are produced in elevated concentrations in psoriasis and obesity. Recently, accumulating evidence shows an increase in OPN levels in both psoriasis and obesity patients. There are strong associations between obesity and psoriasis, the severity of psoriasis increasing with increase in weight. $[22,23]$. In the present study, we confirmed that osteopontin, CXCL9, and CCL5 were higher in patients with psoriasis than in BMI-matched controls. Patients and controls were not age-matched in this work, which may be a drawback. In the present study, we showed, by multivariate logistic regression analysis, that neither age nor disease duration were associated with OPN levels $\geqslant 17.65$.

We confirmed previous observations that OPN plasma levels were enhanced in patients with psoriasis. However, the absence of differences between OPN levels in obese and non-obese psoriasis patients and between obese and non-obese controls suggests that OPN is a factor in psoriasis pathogenesis. We also showed that increases in CXCL9 and CCL5 levels in psoriasis were not affected by obesity or central obesity. Moreover, the absence of a correlation between OPN and TNF or CXCL9 levels suggests that the source of OPN in psoriasis patients may be different than that of TNF and CXCL9.

High serum concentrations of OPN were identified in psoriasis patients and also in obese patients without psoriasis [2,3]. Studies have shown increased plasma levels of OPN in obese versus lean subjects, but no differences were found between obese and overweight patients $[17,26]$. Nevertheless, the result of weight loss on OPN levels is not clear, as different studies have shown conflicting findings [4-7]. Additionally, OPN levels in psoriasis patients may be influenced by the presence of comorbidities; an association between high OPN levels and hypertension or diabetes was observed [27]. Therefore, it is possible that OPN participates in the pathogenesis of inflammatory and metabolic conditions associated with obesity, including non-alcoholic fatty liver disease and atherosclerosis, more than reflecting high BMI per se.

In the present study, using a considerable number of psoriasis patients and obese and non-obese controls, it was observed that OPN was higher in psoriasis patients than in controls, though we did not find an association between obesity and increasing levels of OPN. The absence of a relationship between obesity and OPN may be because its activity is primarily in the adipose tissues and not in the circulation [28].

PsA is an important and severe complication of psoriasis. According to some studies, facial involvement has received little attention and might be a sign of severe psoriasis [24,25,29]. We observed in our cohort $41 \%$ of patients with facial involvement and $13 \%$ with PsA. The identification of biomarkers associated with psoriasis severity is important to predict the natural history of the disease, identification of patients with susceptibility to develop severe disease, and to determine the early use of more effective therapies. Attempts to associate cytokine levels, and more specifically chemokines such as CCL2, CCL3, CXCL1, CXCL9, IL-6, and IL-12 or IL-13, with psoriasis severity have shown contradictory results [14-16]. One study showed that, despite higher levels of CXCL9, CXCL10, CCL17, CCL20, and CCL22 in psoriasis patients than controls, only CCL20 correlated with disease severity and none of these cytokines were affected by clinical improvement after UVB therapy [14]. Another study did not find differences in CXCL9, CXCL10, or CXCL16 between patients and controls, nor correlation with disease severity [30]. IL-1, IL-6, CCL2, CCL3, CCL5, CXCL5, CXCL1, CXCL8, and CXCL9 were evaluated by two different studies, which did not find correlations with disease severity [15,16]. Finally, a meta-analysis evaluated 22 studies on IL-6, and concluded that PASI has no effect on the standardized mean differences in IL-6 [31].

Regarding OPN, one study found a high correlation between OPN and PASI [32], although several studies in psoriasis patients have not found any correlations between plasma OPN and PASI $[27,33,34]$. Two studies evaluated correlations between immunohistochemistry expression of OPN and severity [35,36], but only in one case was a correlation between PASI and OPN documented. However, both studies were performed with small samples. Factors that may contribute to the contradictory findings include age, obesity, and disease duration. In the present study, these confounding factors were considered and no association between PASI and OPN was documented. (a)

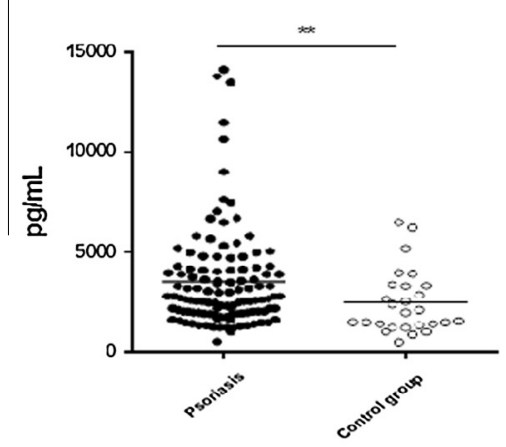

Mann Whitney test Mann Whit
$\mathrm{P}<0.01^{* *}$

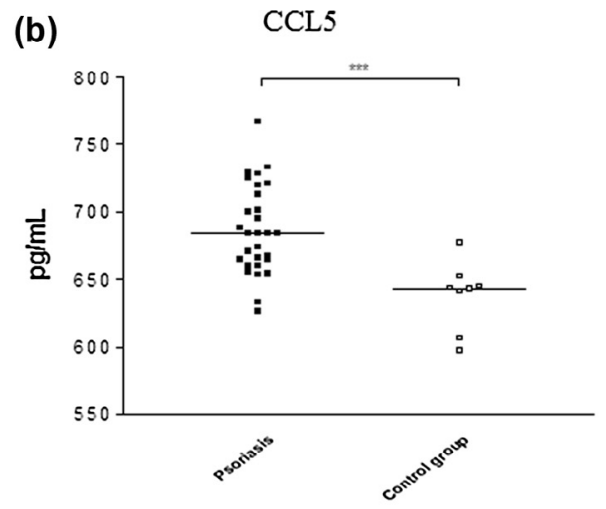

Mann Whitney test Mann Whitne
$\mathrm{P}<0.001^{* * * *}$

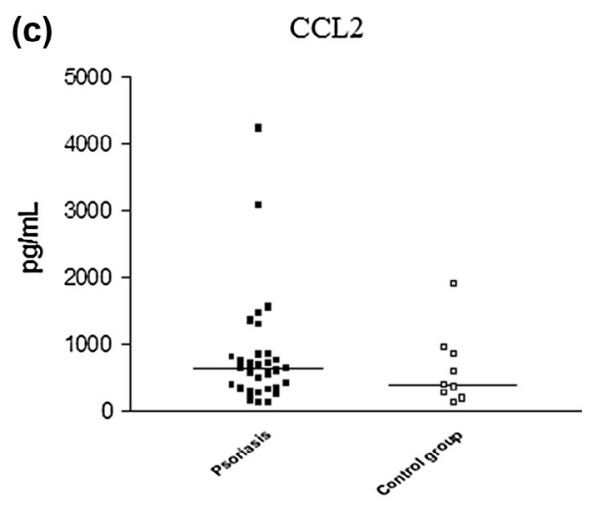

Mann Whitney test $\mathrm{P}>0.05$

Fig. 3. Comparison of CXCL-9 (a), CCL-2 (b), and CCL-5 (c) plasma levels between psoriasis patients and controls. 

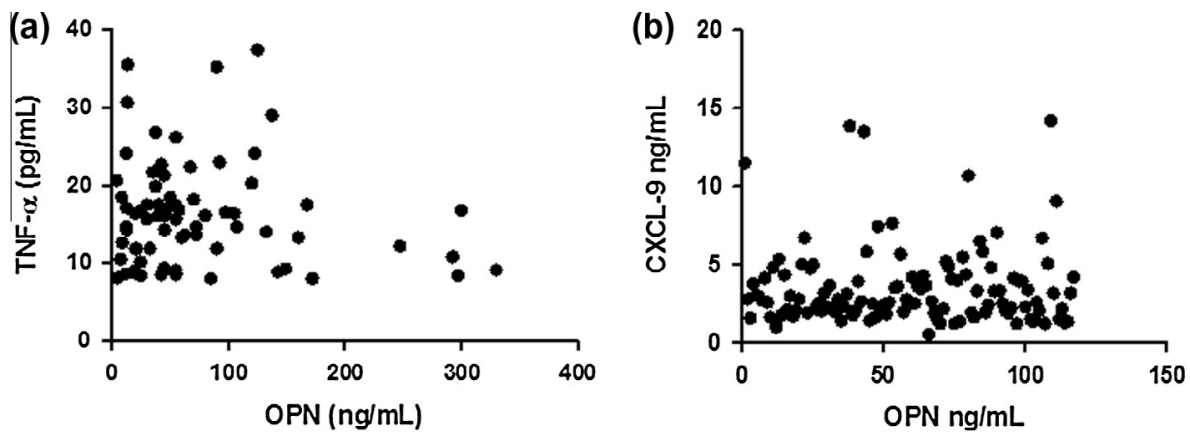

Fig. 4. Correlation between levels of OPN and TNF- $\alpha$ (4a; $P>0.05 ; 95 \% \mathrm{CI}=-0.28$ to 0.18$)$ and $\mathrm{CXCL9}(4 \mathrm{~b} ; P>0.05 ; 95 \% \mathrm{CI}=-0.17$ to 0.19$)$.

Tissue OPN, detected by ELISA, was compared among patients in treatment with methotrexate, cyclosporine, and PUVA and healthy volunteers. Baseline lesional skin OPN levels correlated to PASI. The three therapeutic modalities were associated with significant decreases in the mean levels of PASI and tissue OPN. PUVA achieved the greatest reduction in OPN levels, although it caused the least reduction in PASI; cyclosporine promoted the greatest reduction in PASI, but did not cause the greatest reduction in OPN levels [37]. An indirect correlation with psoriasis severity is the finding that the intensity and quality (nuclear or cytoplasmatic pattern) of OPN expression were modified after PUVA therapy [34].

We found that CCL5 and CXCL9 levels in psoriasis patients were higher than those observed in the control group and not affected by psoriasis severity or obesity. CCL5 is a T lymphocyte chemotactic factor and its increase is in agreement with the pathogenesis of psoriasis $[15,16]$. Studies have shown that CCL2 levels in psoriasis patients are higher than in normal subjects [15,16], promoting angiogenesis and inflammation [16], and reduced after biological therapy [38]. CCL2 is the most extensively studied CC chemokine and has a role in the pathogenesis of obesity and macrovascular complications of diabetes $[39,40]$. Several studies associated high CCL2 levels with atherosclerosis, but it has not yet been proven whether this finding is independent of traditional cardiovascular risk factors, including hypertension [18,41,42]. Atherosclerosis and psoriasis share several pathogenic pathways and, up to now, CCL2 enhancement appears to correlate more strongly in obese individuals than in those of normal body weight [39]. We did not observe differences in CCL2 levels between psoriasis patients and the control group. Our findings may be related to one or both of the following factors: High CCL2 levels detected in obese versus non-obese patients, although these were not confirmed by other anthropometric measures; and/or a small subgroup sample. Further studies addressing CCL2 levels in larger cohorts of BMI-matched psoriasis patients and controls are needed, and the effects of a number of comorbidities must be evaluated to consider CCL2 as an independent psoriasis biomarker. We also highlight that one limitation of CCL5 and CCL2 subgroup analysis is the fewer number of controls.

As psoriasis treatment knowledge has progressed, novel approaches, such as phosphodiesterase 4 inhibitors (PDE4), which inhibit several pro-inflammatory cytokines and chemokines' production simultaneously, have demonstrated the clinical relevance of chemokines [43]. Furthermore, this reinforces the need to understand the chemokine network on psoriasis pathogenesis. As OPN receptors were found in keratinocytes and monocytes [1], it is possible that OPN could enhance cytokine and chemokine production by activating these cells, which could, in turn, enhance Th1 or Th17 polarization. Local expression of OPN in lesional keratinocytes, inflammatory cells, and endothelial cells and its correlation with severity favors the hypothesis that OPN is involved in the pathophysiology of psoriasis [35]. The absence of correlation between plasma OPN and CXCL9 or between plasma OPN and TNF may be related to an independent inflammatory pathway or source of OPN in psoriatic disease and needs to be confirmed by other studies. In contrast to plasma OPN, it is possible that the high correlation of skin OPN with psoriasis severity reflects a local role and a more pronounced action in the epidermal and dermal compartments of psoriatic skin than in circulating T cells. Analogous to macrophages of adipose tissue in obesity and synovial $\mathrm{T}$ cells in rheumatoid synovitis, the overexpression of OPN in psoriasis seems to be correlated to disease severity only in the skin, as suggested by previous studies. The confined reduction of local OPN after systemic therapies or phototherapy may reflect an indirect rather than direct effect of the therapy on OPN levels, once OPN is not proportionally reduced with the decrease of PASI score $[28,37,44]$. Since OPN plasma levels were also reduced after therapy with adalimumab or etanercept [45], further studies addressing OPN levels in non-responder patients treated with anti-TNF- $\alpha$ are necessary to investigate its importance as an alternative inflammatory pathway in psoriasis.

In conclusion, this work emphasizes that OPN, CCL2, CXCL9, and CCL5 plasma levels might not be biomarkers of psoriasis severity, but that OPN, CCL5, and CXCL9 elevation in psoriasis patients is not a consequence of excess weight. Moreover, the absence of a relationship between OPN and the production of inflammatory cytokines and chemokines emphasizes the importance of this molecule in psoriasis pathogenesis and as a psoriasis biomarker.

\section{Financial support}

Financial support for this study was provided by FAPESB Fundação de amparo à pesquisa do estado da Bahia - and Immunology Service of Federal University of Bahia.

\section{Acknowledgment}

We acknowledge Yuri Oliveira and Otávio Nassif for their review assistance.

\section{References}

[1] Zeyda M, Gollinger K, Todoric J, et al. Osteopontin is an activator of human adipose tissue macrophages and directly affects adipocyte function. Endocrinology 2011:152(6):2219-27.

[2] Buback F, Renkl AC, Schulz G, et al. Osteopontin and the skin: multiple emerging roles in cutaneous biology and pathology. Exp Dermatol 2009;18(9):750-9.

[3] Komorowski J, Jankiewicz-Wika J, Kolomecki K, et al. Systemic blood osteopontin, endostatin, and E-selectin concentrations after vertical banding surgery in severely obese adults. Cytokine 2011;55(1):56-61.

[4] Bertola A, Deveaux V, Bonnafous S, et al. Elevated expression of osteopontin may be related to adipose tissue macrophage accumulation and liver steatosis in morbid obesity. Diabetes 2009;58(1):125-33. 
[5] Riedl M, Vila G, Maier C, et al. Plasma osteopontin increases after bariatric surgery and correlates with markers of bone turnover but not with insulin resistance. J Clin Endocrinol Metab 2008;93(6):2307-12.

[6] Schaller G, Aso Y, Schernthaner GH. Increase of osteopontin plasma concentrations after bariatric surgery independent from inflammation and insulin resistance. Obes Surg 2009;19(3):351-6.

[7] You JS, Ji HI, Chang KJ, et al. Serum osteopontin concentration is decreased by exercise-induced fat loss but is not correlated with body fat percentage in obese humans. Mol Med Rep 2013;8(2):579-84.

[8] Sandberg M, Frykman A, Ernerudh J, et al. Cord blood cytokines and chemokines and development of allergic disease. Pediatr Allergy Im-munol 2009;20:519-27.

[9] Ritchlin CT, Qureshi AA, De Vlam K, et al. Biomarkers in psoriasis and psoriatic arthritis: GRAPPA 2008. J Rheumatol 2010;37(2):462-7.

[10] Giustizieri ML, Mascia F, Frezzolini A, et al. Keratinocytes from patients with atopic dermatitis and psoriasis show a distinct chemokine production profile in response to $\mathrm{T}$ cell-derived cytokines. J Allergy Clin Immunol 2001; 107:871-7.

[11] Wilsmann-Theis D, Hagemann T, Jordan J, et al. Psoriasis and atopic dermatitis: are there more similarities or more differences? Eur J Dermatol 2008;18(2):172-80.

[12] Flier J, Boorsma DM, van Beek PJ, et al. Differential expression of CXCR3 targeting chemokines CXCL10, CXCL9, and CXCL11 in different types of skin inflammation. J Pathol 2001;194:398-405.

[13] Stoof TJ, Filer J, Sampat S, et al. The antipsoriatic drug dimethylfumarate strongly suppresses chemokine production in human keratinocytes and peripheral blood mononuclear cells. Br J Dermatol 2001;144(6):1114-20.

[14] Ekman AK, Sigurdardottir G, Carlström M, et al. Systemically elevated Th1-, Th2- and Th17-associated chemokines in psoriasis vulgaris before and after ultraviolet B treatment. Acta DermVenereol 2013;93(5):527-31.

[15] Johnston A, Arnadottir S, Gudjonsson JE, et al. Obesity in psoriasis: leptin and resistin as mediators of cutaneous inflammation. $\mathrm{Br} J$ Dermatol 2008;159(2):342-50.

[16] Pohl D, Andrýs C, Borská L. CC and CXC chemokines patterns in psoriasis determined by protein array method were influenced by Goeckerman's therapy. Acta Medica (Hradec Kralove) 2009;52(1):9-13.

[17] Ahmad R, Al-Mass A, Al-Ghawas D, et al. Interaction of osteopontin with IL-18 in obese individuals: implications for insulin resistance. PLoS One13 2013;8(5):e63944.

[18] Ohmori R, Momiyama Y, Taniguchi H, et al. Plasma osteopontin levels are associated with the presence and extent of coronary artery disease. Atherosclerosis 2003;170:333-7.

[19] Lee BC. Lee J (2014) Cellular and molecular players in adipose tissue inflammation in the development of obesity-induced insulin resistance. Biochim Biophys Acta 1842;3:446-62.

[20] Surmi BK, Hasty AH. The role of chemokines in recruitment of immune cells to the artery wall and adipose tissue. Vascul Pharmacol 2010;52(1-2):27-36.

[21] Maksymowych WP, Fitzgerald O, Wells GA, et al. Proposal for levels of evidence schema for validation of a soluble biomarker reflecting damage endpoints in rheumatoid arthritis, psoriatic arthritis, and ankylosing spondylitis, and recommendations for study design. J Rheumatol 2009;36(8):1792-9.

[22] Duarte GV, de Oliveira MF, Cardoso TM, et al. Association between obesity measured by different parameters and severity of psoriasis. Int J Dermatol 2013;52(2):177-81.

[23] Takahashi H, Tsuji H, Takahashi I, et al. Prevalence of obesity/adiposity in Japanese psoriasis patients: adiposity is correlated with the severity of psoriasis. J Dermatol Sci 2009;55(1):74-6.
[24] Canpolat F, Cemil BC, Eskioğlu F, et al. Is facial involvement a sign of severe psoriasis? Eur J Dermatol 2008;18(2):169-71.

[25] Park JY, Rim JH, Choe YB. Facial psoriasis: comparison of patients with and without facial involvement. J Am Acad Dermatol 2004;50(4):582-4.

[26] Gómez-Ambrosi J, Catalán V, Ramírez B, et al. Plasma osteopontin levels and expression in adipose tissue are increased in obesity. J Clin Endocrinol Metab 2007;92(9):3719-27.

[27] Chen YJ, Shen JL, Wu CY, et al. Elevated plasma osteopontin level is associated with occurrence of psoriasis and is an unfavorable cardiovascular risk factor in patients with psoriasis. J Am Acad Dermatol 2009;60(2):225-30.

[28] Kiefer FW, Zeyda M, Todoric J, et al. Osteopontin expression in human and murine obesity: extensive local up-regulation in adipose tissue but minimal systemic alterations. Endocrinology 2008;149(3):1350-7.

[29] Woo SM, Choi JW, Yoon HS, et al. Classification of facial psoriasis based on the distributions of facial lesions. J Am Acad Dermatol 2008;58(6):959-63.

[30] Lima XT, Oliveira RT, Braga FG, et al. Circulating levels of chemokines in psoriasis. Autoimmunity $2015 \mathrm{Feb}$;48(1):57-60.

[31] Dowlatshahi EA, van der Voort EA, Arends LR, et al. Markers of systemic inflammation in psoriasis: a systematic review and meta-analysis. $\mathrm{Br}$ Dermatol 2013;169(2):266-82.

[32] Kadry D, Hegazy RA, Rashed L. Osteopontin and adiponectin: how far are they related in the complexity of psoriasis? Arch Dermatol Res 2013;305(10):939-44.

[33] Buommino E, Tufano MA, Balato N, et al. Osteopontin: a new emerging role in psoriasis. Arch Dermatol Res 2009;301(6):397-404.

[34] Erturkler E, Cicek D, Kaman D, et al. Plasma osteopontin levels in patients with Behcet's disease and psoriasis. Eur J Dermatol 2011;21(2):203-8.

[35] Abdou AG, El Farargy S, Seleit I, et al. Osteopontin expression in chronic plaque psoriasis: an association with the severity of disease. Anal Quant Cytol Histol 2012;34(2):79-85.

[36] Amin M, Azim ZA. Immunohistochemical study of osteopontin, Ki-67, and CD34 of psoriasis in Mansoura, Egypt. Indian J Pathol Microbiol $2012 \cdot 55(1): 56-60$

[37] El-Eishi NH, Kadry D, Hegazy RA, et al. Estimation of tissue osteopontin levels before and after different traditional therapeutic modalities in psoriatic patients. J Eur Acad Dermatol Venereol 2013;27(3):351-5.

[38] Lembo S, Capasso R, Balato A, et al. MCP-1 in psoriatic patients: effect of biological therapy. J Dermatol Treat 2014;25(1):83-6.

[39] Panee J. Monocyte chemoattractant protein 1 (MCP-1) in obesity and diabetes. Cytokine 2012;60(1):1-12.

[40] Sozer V, Himmetoglu S, Korkmaz GG, et al. Paraoxonase, oxidized low density lipoprotein, monocyte chemoattractant protein-1 and adhesion molecules are associated with macrovascular complications in patients with type 2 diabetes mellitus. Minerva Med. 105(3):237-44.

[41] Coll B, Alonso-Villaverde C, Joven J. Monocyte chemoattractant protein-1 and atherosclerosis: is there room for an additional biomarker? Clin Chim Acta 2007;383(1-2):21-9.

[42] Martynowicz H, Janus A, Nowacki D, et al. The role of chemokines in hypertension. Adv Clin Exp Med 2014;23(3):319-25.

[43] Schafer PH, Day RM. Novel systemic drugs for psoriasis: mechanism of action for apremilast, a specific inhibitor of PDE4. J Am Acad Dermatol 2013;68(6):1041-2.

[44] Xu G, Nie H, Li N, et al. Role of osteopontin in amplification and perpetuation of rheumatoid synovitis. J Clin Invest 2005;115(4):1060-7.

[45] Buommino E, De Filippis A, Gaudiello F, et al. Modification of osteopontin and MMP-9 levels in patients with psoriasis on anti-TNF- $\alpha$ therapy. Arch Dermato Res 2012;304(6):481-5. 